\title{
Caracterización ficológica en manantiales de la cuenca baja del sistema hidrológico del Pánuco, México
}

\author{
JAVIER CARMONA JimÉNEZ y GUSTAVO MONTEJANO ZURITA ${ }^{1}$
}

\begin{abstract}
RESUMEN. Se presentan los resultados del estudio ficoflorístico de tres manantiales en la región de la Huasteca Potosina, en el que se reportan 67 especies algales. Se analizan las diferencias y similitudes florísticas y su relación con algunos parámetros ambientales como la iluminación y velocidad de corriente, que fueron los factores más importantes que afectaron las formas de crecimiento predominantes en estos ambientes. En estos manantiales se definieron los microambientes y las principales asociaciones algales que los caracterizan.
\end{abstract}

ABSTRACT. The results of a phycofloristic study on three springs in the Huasteca Potosina region are presented; 67 species are reported. Floristic similarities and differences among the springsare analized, as well as the relationship between species and enviromental factors. Microhabitas were defined in the springs as well as the principal algal asociations that characterize them. Light intensity and current velocity are the most important factors affecting the predominant growth forms present in this type of habitat.

Los manantiales presentan características distintas de otros ambientes lóticos y han sido considerados por Hynes (1970), como ambientes acuáticos especiales. Entre los factores que los distinguen de otros ambientes podemos mencionar las siguientes: el aporte relativamente constante del volumen de agua, escasa variación en la composición química de ésta, temperatura constante y bajas concentraciones de materia orgánica. Además, es común que existan gradientes de iluminación cuando se originan dentro de cuevas o cavidades y por la cobertura de la vegetación. Esta característica de estabilidad en los manantiales contrasta con los cambios bruscos que ocurren en el cauce de un río.

A nivel mundial la mayoría de los estudios ficológicos en estos ambientes están referidos a manantiales termales y sólo unos cuantos a manantiales llamados fríos o de

${ }^{1}$ Laboratorio de Ficología, Facultad de Ciencias, Universidad Nacional Autónoma de México, Apdo. Postal 70-620. Delegación Coyoacán, 04510. México, D.F. 
infiltración. De estos últimos trabajos cabe destacar los realizados en Europa y Estados Unidos por Whitford, 1956; Round, 1957c, 1960c, citados en Margaleff (1977); Golubic, 1967, Dary y Wayne, 1968, Hynes, 1970, y Round, 1973, 1984.

En México existen algunos registros de algas para manantiales termales en Baja California Sur, Oaxaca, Hidalgo y Edo. de México (Sámano, 1932; Ortega, 1984), y algunas referencias de la presencia de especies que habitan en manantiales de Morelos, Puebla y Oaxaca, aunque no se menciona el tipo de manantial, ni son trabajos específicos sobre el ambiente (Sokoloff, 1936; Ortega, 1984).

El trabajo pretende caracterizar la ficoflora de los manantiales y conocer las condiciones ambientales que afectan su manifestación, lo cual requiere del estudio de las condiciones microambientales. Se presentan los resultados del estudio de las algas de tres manantiales de la Huasteca Potosina, dos de ellos afluentes al río Tampaón (nacimiento del río Choy y nacimiento de Puente de Dios) y uno afluente del río Moctezuma (nacimiento del río Huichihuayan), que es colector principal de la cuenca del Río Pánuco.

\section{ÁREA DE ESTUDIO}

La Cuenca del Río Pánuco es una de las regiones hidrográficas más importantes del país, tanto por la superficie que ocupa ( $84^{\prime} 956 \mathrm{~km}^{2}$ ), situada en cuarto lugar de la República Mexicana, como por el volumen de sus escurrimientos, ya que ocupa el quinto lugar (Secretaría de Recursos Hidráulicos, 1971). Está dividida en dos porciones: alto y bajo Pánuco. Los manantiales de estudio de ubican en el bajo Pánuco que está formado por las cuencas de los ríos Extóraz, Bajo Amajac, Tamasopo, Tempoal, Tampaón, Moctezuma y Pánuco, las cuales recorren la mayor parte del estado de San Luis Potosí correspondientes a la región llamada Huasteca Potosina. (fig. 1).

El clima de la región es cálido subhúmedo con lluvias en verano; su temperatura media anual oscila entre los $27^{\circ} \mathrm{C}$ y $29^{\circ} \mathrm{C}$ y la media mensual que fluctúa entre $28^{\circ} \mathrm{C}$ y $32^{\circ} \mathrm{C}$. La precipitación media anual varía de los 848 a los $1062 \mathrm{~mm}$. (Instituto Nacional de Estadística Geografía e Informática, 1985).

Conforme al Instituto Nacional de Estadística Geografía e Informática (1985), los tres manantiales fueron considerados de infiltración, donde el agua que emana se origina de las precipitaciones atmosféricas. De acuerdo a la temperatura del agua figuran como manantiales ordinarios o isotérmicos (Vasiliev y Arabadzhi, 1981). El manantial Choy (fig. 1) está localizado a los $98^{\circ} 48^{\prime} 09^{\prime \prime}$ longitud oeste y los $21^{\circ} 59^{\prime} 42^{\prime \prime}$ latitud norte. Nace a una elevación de $50 \mathrm{msnm}, 17 \mathrm{Km}$ al norponiente de Tamuín. El curso general del río es suroriente y confluye con el río Tampaón. El escurrimiento medio anual del manantial es de $156 \times 10 \mathrm{~m}^{3}$ (Secretaría de Recursos Hidráulicos, 1971). Es un manantial de grandes dimensiones que consta de un borbollón que se origina del subsuelo dentro de una caverna de $20 \mathrm{~m}$ de altura por 15 a $20 \mathrm{~m}$ de ancho y $20 \mathrm{~m}$ de profundidad. En el techo 


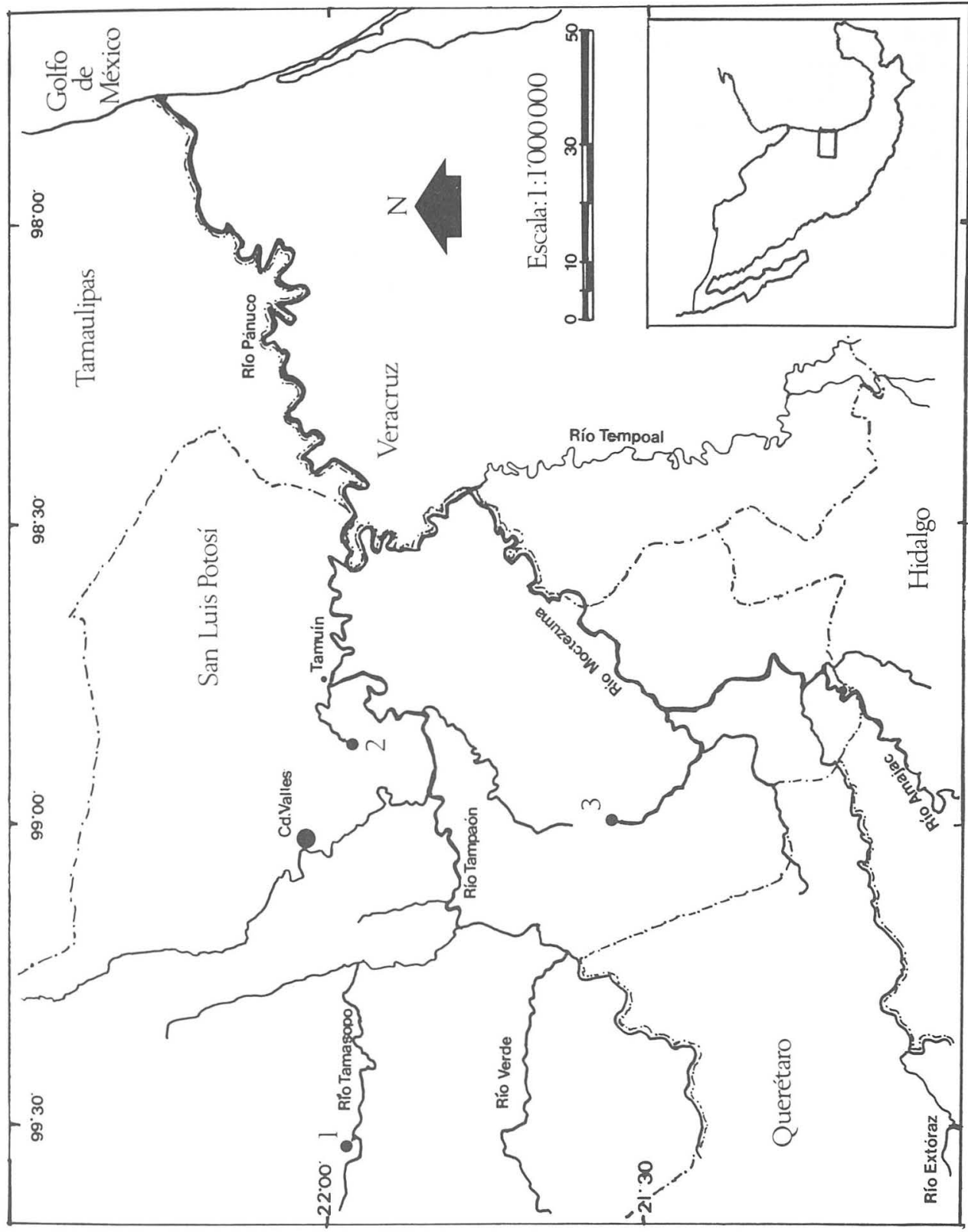

Fig.1 Mapa de localidades. Ubicación de los manantiales de estudio en la Huasteca Potosina, México. 1, Puente de Dios. 2, Choy. 3, Huichihuayan. 
de la caverna se abre un boquete de $5 \mathrm{~m}$ aproximadamente de diámetro que permite que se ilumine la caverna. El flujo de agua sale de la caverna después de recorrer un pasillo de $25 \mathrm{~m}$ de largo iniciando lo que propiamente es el río. El manantial Huichihuayan (fig. 1) se localiza a los $98^{\circ} 56^{\prime} 10^{\prime \prime}$ longitud oeste y los $21^{\circ} 33^{\prime} 15^{\prime \prime}$ latitud norte. Nace a una altitud de $100 \mathrm{msnm}$. El río Huichihuayan recorre $25 \mathrm{~km}$ antes de unirse con el río Moctezuma. El manantial se encuentra dentro de una caverna que en la zona de la entrada tiene de abertura $5 \mathrm{~m}$ de alto por $10 \mathrm{~m}$ de ancho y $2 \mathrm{~m}$ de profundidad. El cauce de la poza se comunica con "canales de corriente" ${ }^{\prime 2}$ con amplia cobertura de vegetación, y por los cuales se inicia lo que practicamente es el río.

El manantial Puente de Dios (fig. 1) es uno de los afluentes del río Tamasopo, el cual recorre $100 \mathrm{~m}$ antes de unirse con el río. Está localizado a los $99^{\circ} 33^{\prime} 19^{\prime \prime}$ longitud oeste y los $21^{\circ} 55^{\prime} 15^{\prime \prime}$ latitud norte. Nace a una elevación de $450 \mathrm{msnm}$.

El nacimiento está constituído de una caverna de $10 \mathrm{~m}$ de ancho, $2.5 \mathrm{~m}$ de alto y no más de $0.50 \mathrm{~m}$ de profundidad. El techo de la caverna vá disminuyendo hasta desaparecer en el nivel del agua. El cauce del manantial se continúa en un arroyo muy sombreado por la cobertura de la vegetación.

\section{Metodología}

Con el objeto de reconocer microambientes dentro de los manantiales estudiados, se llevó a cabo una sectorización preliminar de la localidad, empleando como criterio la homogeneidad, al menos aparente, de los siguientes aspectos: las formas de crecimiento algales, la fisiografía del terreno, el sustrato, la insolación y la velocidad de corriente. A esta primera aproximación desarrollada en la colecta la denominamos "zona", y una vez confirmada por el análisis de la composición algal la consideramos microambiente. La insolación la empleamos como una estimación de la cantidad de luz que llega al punto de colecta y es expresada como el porcentaje del horizonte que se observa desde el punto de colecta (Johansson 1982), y que se expresa en este trabajo en los siguientes términos: insolación muy alta $=>80 \%$ del horizonte visible; alta $=60-80 \%$; media $=40-60 \%$; baja $=20-40 \%$ y muy baja $=<20 \%$. Además, se tomaron datos de intensidad luminosa para varios puntos de colecta, aunque estos no se emplearon en la sectorización preliminar, debido a su variación con la hora del día y la nubosidad.

Para la velocidad de corriente se tomaron dos tipos de datos, unos cualitativos y otros cuantitativos. Para las colectas de los meses del año 1989, la mayor parte de los datos se obtuvo empleando un medidor de corriente Swoffer Instruments Modelo 2100 (Series Current Velocity Meters). Sin embargo, en varios puntos donde no se pudo

${ }^{2}$ En este estudio "canal de corriente" se entiende por la zona donde el cauce del río se reduce y acelera considerablemente su velocidad. 
emplear el medidor ( $v$.gr. escasa profundidad) y en las colectas de los años anteriores a 1989, se tomaron datos estimativos tomando como base las cinco categorias empleadas por Johansson (1982): muy baja (casi estancada), baja, moderada, alta y muy alta. Con el propósito de poder relacionar los dos tipos de datos se estableció la siguiente correspondencia, basada en los valores mínimos y máximos registrados en el área de estudio y en mediciones llevadas en el campo con este objetivo: $<0.1 \mathrm{~m} / \mathrm{seg}=$ muy baja; $0.1-0.35 \mathrm{~m} / \mathrm{seg}=$ lenta; $0.35-0.6 \mathrm{~m} / \mathrm{seg}=\operatorname{moderada} ; 0.6-0.85 \mathrm{~m} / \mathrm{seg}=$ alta y $>0.85 \mathrm{~m} / \mathrm{seg}=$ muy alta. Para la descripción de las formas de crecimiento se tomó como punto de partida los trabajos de Holmes y Whitton (1981) y Tavera y GonzálezGonzález (1990). Con base en los criterios de sectorización mecionados encontramos lo siguiente:

En el nacimiento del Choy se delimitaron tres zonas: (fig.2,A)

Zona 1. Canales de corriente dentro de la caverna. Formas de crecimiento predominantes: costras y tufos. Intensidad luminosa baja. Velocidad de corriente muy alta. Es la zona donde fluye la mayor parte del cauce del manantial. Sustrato, rocas ígneas y calcáreas de diámetro considerable (1 m o más) que llegan a emerger a la superficie del agua.

Zona 2. Cantos rodados dentro de la caverna. Formas de crecimiento predominantes: costras, flóculos filamentosos y musgos, sobre los cuales se desarrollan algas. Iluminación baja y velocidad de corriente moderada. Sustrato, cantos rodados y guijarros de roca ígnea y calcárea a una profundidad de $0.50 \mathrm{~m}$.

Zona 3. Rocas emergentes fuera de la caverna. Grandes rocas de hasta $3 \mathrm{~m}$ de diámetro. Formas de crecimiento predominantes: costras, flóculos filamentosos, tapetes, en los cuales no se distingue una trama de filamentos, talos filiformes y crecimientos sobre musgos. Intensidad luminosa media. Velocidad de corriente moderada. Sustrato, rocas emergentes, ígneas y calcáreas, donde la profundidad es de hasta $3 \mathrm{~m}$.

En la localidad Huichihuayan se delimitaron seis zonas: (fig.2,B)

Zona 1. Rocas emergentes en el afloramiento del agua dentro de la caverna. Formas de crecimiento pedominantes: costras, flóculos filamentosos, tufos y musgos. Intensidad luminosa muy baja. Velocidad de corriente lenta. Sustrato, formado por rocas emergentes de 1 a $5 \mathrm{~m}$ de diámetro. Profundidad 1 a $1.5 \mathrm{~m}$.

Zona 2. Canales de corriente que se forman después de emerger el agua de la caverna. Formas de crecimiento predominantes: costras, tapetes, tufos, y flóculos filamentosos. Intensidad luminosa media. Velocidad de corriente muy alta. Sustrato, roca ígnea. Profundidad, 0.50 a $1 \mathrm{~m}$.

Zona 3. Cantos rodados pequeños, en el centro del cauce del río fuera de la caverna. Formas de crecimiento prediminantes: costras, tapetes y flóculos filamentosos. Intensidad luminosa muy alta. Velocidad de corriente lenta. Sustrato, cantos rodados. Profundidad, 0.5 a $1 \mathrm{~m}$. 

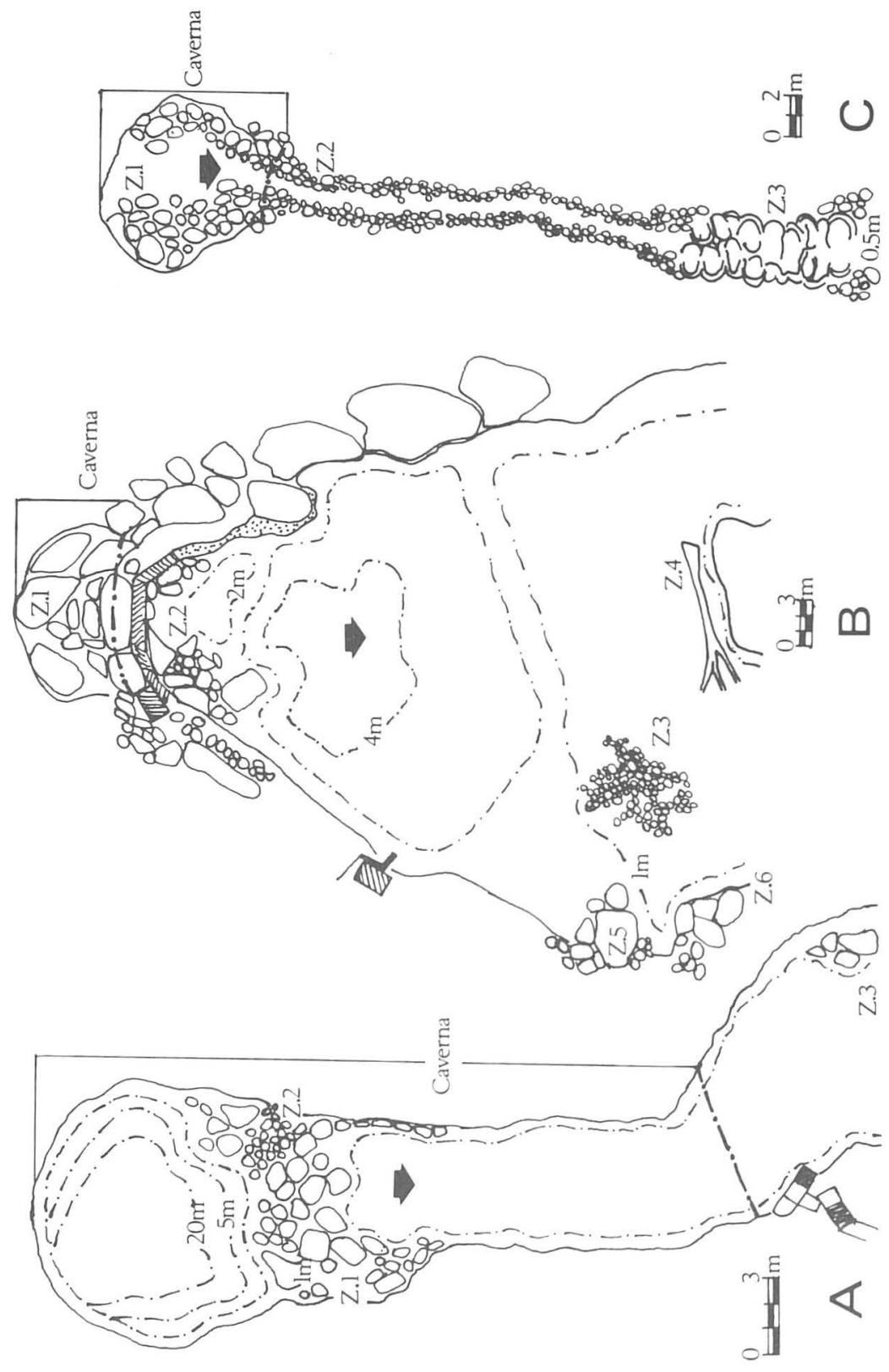

Fig.2 Zonas de colecta en los manantiales de estudio. A, Choy. B, Huichihuayan. C, Puente de Dios. 
Zona 4. Islote con troncos fuera de la caverna en el centro del cauce del río, además, donde se desarrollaron y observaron crecimientos algales. Formas de crecimiento predominantes: tapetes, flóculos filamentosos y colchones. Intesidad luminosa alta. Velocidad de corriente lenta. Sustrato, sobre troncos. Profundidad, 0.20 a $1 \mathrm{~m}$.

Zona 5. Márgen del río, fuera de la caverna, se observan rocas emergentes de diámetro considerable ( $2 \mathrm{~m}$ o más). Formas de crecimiento predominantes: tapetes, flóculos filamentosos y colchones. Intensidad luminosa media. Velocidad de corriente lenta. Sustrato, rocas ígneas sumergidas desde el nivel del agua hasta $1 \mathrm{~m}$ de profundidad.

Zona 6. Plataformas de rocas sumergidas al márgen del río fuera de la caverna. Formas de crecimiento prediminantes: tapetes y flóculos filamentosos. Intensidad luminosa alta. Velocidad de corriente lenta. Sustrato, roca ígnea. Profundidad, $0.50 \mathrm{~m}$ a $1 \mathrm{~m}$.

En el nacimiento de Puente de Dios se reconocieron tres zonas: (fig.2,C)

Zona 1. Pequeñas rocas sumergidas dentro de la caverna. Forma de crecimiento predominante: costroso. Intensidad luminosa muy baja. Velocidad de corriente lenta. Sustrato de fragmentos de roca ígnea. La profundidad no rebasa los $0.40 \mathrm{~m}$.

Zona 2. Canales de corriente formados por rocas de tamaño variable a la salida de la cueva. Forma de crecimiento predominante: costroso. Intensidad luminosa baja. Velocidad de corriente moderada. Sustrato, roca ígnea. La profundidad varía de $0.10 \mathrm{a}$ $0.50 \mathrm{~m}$.

Zona 3. Repisas o pocitas. Sistema que se forma unos $50 \mathrm{~m}$ después de la caverna. Formas de crecimiento predominantes: costroso, flóculos filamentosos, colchones, talos filiformes, y masas de filamentos flotantes. Intensidad luminosa alta. Velocidad de corriente lenta sin llegar a estancarse pues existe una contínua comunicación entre las pozas. Sustrato roca calcárea y travertinos. La profundidad varía de acuerdo a cada poza, aunque no rebasa $1 \mathrm{~m}$.

En cada zona se tomaron muestras de los crecimientos algales, ya sea tomando fragmentos de roca con cincel y martillo o bién empleando una espátula. Cada muestra fué fijada con formol al $4 \%$ con agua de la localidad e incorporada a la sección ficológica del Herbario de la Facultad de Ciencias, UNAM (FCME) con las siglas PA, correspondientes al proyecto Flora Ficológica de la Cuenca del Río Pánuco.

En el caso de las Cyanophyceae se utilizó el esquema de clasificación de Anagnostidis y Komárek $(1985,1988)$ y Komárek y Anagnostidis (1986, 1989). Para la identificacion de las especies además se emplearon los siguientes trabajos: Geitler (1932); Desikachary (1959); Starmach (1966); Kann y Komárek (1970); Komárek y Kann (1973); Komárek y Kovacik (1987); Watanabe y Komárek (1989). Para el resto de las clases se empleó el esquema genérico de Bourrelly $(1968,1970$ y 1972) y para la identificación específica se emplearon los siguientes trabajos: Rhodophyceae, Israelson (1942); Kylin (1956); Bishoff (1965); Starmach (1977). Xanthophyceae, Ventakataraman (1961); Chlorophyceae, West y West (1904); Transeau (1951); van Den Hoek (1963); Islam 
(1963); Starmach (1969); Bacillariophyceae, Patrick y Reimer (1966, 1975); Germain (1981); Sarode y Kamat (1984).

\section{RESUltados}

Dentro de cada uno de los manantiales los factores que más variaron fueron la iluminación, velocidad de corriente y el sustrato. La interacción de estos factores estuvo relacionada con la presencia de diferentes asociaciones y formas de crecimiento. A esta coincidencia de factores y asociaciones es a lo que denominamos microambientes. Las características de cada microambiente se presentan en el cuadro 1 . Se identificaron un total de 67 especies de algas, 31 pertenecientes a la clase Bacillariophyceae (46.26\%), 17 a la Cyanophyceae (25.37\%), 14 a la Chlorophyceae (20.89\%), 3 a la Rhodophyceae (4.47\%) y 2 a la Xantophyceae (2.98\%). El total de especies y el porcentaje por clase de cada manantial se presenta en el cuadro 2.

La falta de epíteto específico en varias especies se debió a dos razones: a la falta de estructuras reproductoras necesarias para su identificación, como en el caso de las Zygnematales (Chlorophyceae); o bién a que las características taxonómicas no coinciden con las de especies previamente descritas, como en el caso de las Cyanophyceae. En ambos casos, cuando se presentaron diferencias en características taxonómicas en dos o mas poblaciones, las consideramos provisionalmente como especies diferentes (por ejemplo: Cladophora sp.1 y Cladophora sp.2). La distribución de las especies por manantial se presenta en los cuadros 3, 4 y 5 . Algunas de estas especies se presentaron de forma recurrente espacial y temporalmente en los manantiales, bajo condiciones ambientales similares, por lo que las consideramos asociaciones, en el sentido empleado por Hutchinson (1967). Dos de estas asociaciones, se presentaron en las tres colectas bajo condiciones ambientales particulares en al menos dos localidades. La asociación Hildenbrandia rivularis, Hyella fontana y Pleurocapsa minor, con forma de crecimiento costroso, se presentó en el microambiente 2 del manantial Choy y el microambiente 1 del manantial Puente de Dios. En todos los casos creciendo bajo condiciones de iluminación baja y muy baja (645-2000 luxes), y velocidad de corriente moderada y lenta (0.1-0.3 m/seg).

La asociación Hildenbrandia rivularis, Audouinella sp. se encontró con forma de crecimiento costroso y tufos, en los microambientes 1 del manantial Choy y el microambiente 2 del manantial Huichihuayan, bajo condiciones de intensidad luminosa media y baja (250-1000 luxes) y velocidad de corriente alta y muy alta (0.6-1.3 m/seg). Asimismo, algunas especies se pesentaron en las tres colectas pero solo en una localidad. Tal es el caso de Cladophora sp.2, Audouinella sp., Achnanthes inflata y Cocconeis placentula, en el microambiente 1 del manantial Huichihuayan; Merismopedia glauca, Amphipleura pellucida y Gyrosigma scalproides .n el microambiente 4 del manantial Huichihuayan; 
CUADRO 1. Características físicas, químicas y biológicas de los microambientes en los manantiales de estudio

\begin{tabular}{|c|c|c|c|c|c|c|c|c|c|c|c|}
\hline Localidad & $\begin{array}{l}\text { Fecha de } \\
\text { colecta } \\
\text { y/o estación }\end{array}$ & Microambiente & Nombre & Clima ${ }^{a}$ & $\mathrm{~T}^{\circ} \mathrm{C}$ & $\mathrm{pH}$ & $\begin{array}{l}\text { intensidad } \\
\text { Luminosa }^{\mathrm{b}}\end{array}$ & $\begin{array}{l}\text { Velocidad } \\
\text { de corriente }^{c}\end{array}$ & $\begin{array}{l}\text { Profundidad } \\
\text { (m) }\end{array}$ & Sustrato & $\begin{array}{l}\text { forma de } \\
\text { crecimiento }{ }^{d}\end{array}$ \\
\hline \multirow[t]{5}{*}{$\begin{array}{l}\text { Manantial } \\
\text { Choy }\end{array}$} & $\begin{array}{l}\text { 13-IV-88 } \\
\text { Secas }\end{array}$ & 1 & $\begin{array}{l}\text { canales de } \\
\text { corriente }\end{array}$ & Aw 1 & 27 & 7 & baja & muy alta & 1 & $\begin{array}{l}\text { ígneo y } \\
\text { calcáreo }\end{array}$ & $\mathrm{c}, \mathrm{t}$ \\
\hline & & 2 & $\begin{array}{l}\text { cantos } \\
\text { rodados }\end{array}$ & " & " & $"$ & $"$ & moderada & 0.5 & $"$ & $\mathrm{c}, \mathrm{m}, \mathrm{fl}$ \\
\hline & & 3 & $\begin{array}{l}\text { rocas } \\
\text { emergentes }\end{array}$ & $"$ & $"$ & $"$ & media & $"$ & 1.5 & $"$ & $\mathrm{fl}$ \\
\hline & $\begin{array}{l}14 / V / 88 \\
\text { Secas }\end{array}$ & 1 & $\begin{array}{l}\text { canales de } \\
\text { corrientes }\end{array}$ & $"$ & 26 & 7.6 & baja & 1 & 1 & $"$ & $\mathrm{c}, \mathrm{t}$ \\
\hline & & 2 & $\begin{array}{l}\text { cantos } \\
\text { rodados }\end{array}$ & $"$ & $"$ & $"$ & 0.4 & 0.5 & $"$ & $"$ & $\mathrm{~m}, \mathrm{fl}$ \\
\hline & & 3 & $\begin{array}{l}\text { rocas } \\
\text { emergentes }\end{array}$ & $"$ & $"$ & $"$ & media & 0.3 & 1.5 & $"$ & fl \\
\hline & $\begin{array}{l}9 / X 1 / 89 \\
\text { Lluvias }\end{array}$ & 1 & $\begin{array}{l}\text { canales de } \\
\text { corriente }\end{array}$ & $"$ & $"$ & $"$ & baja & 1.3 & 1 & $"$ & $c, t$ \\
\hline & & 2 & $\begin{array}{l}\text { cantos } \\
\text { rodados }\end{array}$ & $"$ & $"$ & $"$ & $"$ & 0.6 & 0.5 & $"$ & $\mathrm{~m}, \mathrm{fl}$ \\
\hline & & 3 & $\begin{array}{l}\text { rocas } \\
\text { emergentes }\end{array}$ & $"$ & $"$ & $"$ & media & 0.4 & 1.5 & $"$ & $\mathrm{~m}$ \\
\hline
\end{tabular}


CUADRO 1. Continuación.

\begin{tabular}{|c|c|c|c|c|c|c|c|c|c|c|c|}
\hline Localidad & $\begin{array}{l}\text { Fecha de } \\
\text { colecta } \\
\text { y/o estación }\end{array}$ & Microambiente & Nombre & Clima $^{a}$ & $\mathrm{~T}^{\circ} \mathrm{C}$ & $\mathrm{pH}$ & $\begin{array}{l}\text { intensidad } \\
\text { Luminosa }^{\mathrm{b}}\end{array}$ & $\begin{array}{l}\text { Velocidad } \\
\text { de corriente }^{c}\end{array}$ & $\begin{array}{l}\text { Profundidad } \\
\text { (m) }\end{array}$ & Sustrato & $\begin{array}{l}\text { forma de } \\
\text { crecimiento }^{d}\end{array}$ \\
\hline \multirow[t]{6}{*}{$\begin{array}{l}\text { Manantial } \\
\text { Huichihuayan }\end{array}$} & $\begin{array}{l}11 / \text { III/84 } \\
\text { Secas }\end{array}$ & 1 & $\begin{array}{l}\text { rocas } \\
\text { sumergidas }\end{array}$ & $(\mathrm{A}) \mathrm{C}(\mathrm{m})(\mathrm{w})$ & 21 & 7 & muy baja & lenta & 1 & ígneo & $\mathrm{m} . \mathrm{fl}$ \\
\hline & & 2 & $\begin{array}{l}\text { canales } \\
\text { de comiente }\end{array}$ & $"$ & $"$ & $"$ & media & muy alta & 0.5 & $"$ & $\begin{array}{l}\mathrm{c}, \mathrm{t}, \mathrm{m} \\
\text { fl,ta }\end{array}$ \\
\hline & & 3 & $\begin{array}{l}\text { cantos } \\
\text { rodados }\end{array}$ & $"$ & 21.5 & $"$ & muy alta & lenta & $"$ & $"$ & $\begin{array}{l}\mathrm{c}, \mathrm{fl}, \\
\text { ta }\end{array}$ \\
\hline & & 4 & $\begin{array}{l}\text { islote con } \\
\text { troncos }\end{array}$ & $"$ & $"$ & $"$ & alta & $"$ & 0.2 & troncos & $\begin{array}{l}\mathrm{c}, \mathrm{fl}, \\
\text { ta }\end{array}$ \\
\hline & & 5 & $\begin{array}{l}\text { márgen } \\
\text { del río }\end{array}$ & $"$ & $"$ & $"$ & media & $"$ & 0.5 & ígneo & $\begin{array}{l}\text { cl.fl, } \\
\text { ta }\end{array}$ \\
\hline & & 6 & plataforma & $"$ & $"$ & $"$ & alta & $"$ & $"$ & $"$ & $\mathrm{cl}, \mathrm{fl}$ \\
\hline \multirow[t]{6}{*}{$\begin{array}{l}\text { Manatial } \\
\text { Huichihuayan }\end{array}$} & $\begin{array}{l}2 / V / 85 \\
\text { Secas }\end{array}$ & 1 & $\begin{array}{l}\text { rocas } \\
\text { sumergidas }\end{array}$ & $(\mathrm{A}) \mathrm{C}(\mathrm{m})(\mathrm{w})$ & 21 & 7 & muy baja & lenta & 1 & ígneo & $\mathrm{m} . \mathrm{fl}$ \\
\hline & & 2 & $\begin{array}{l}\text { canales } \\
\text { de corriente }\end{array}$ & $"$ & $"$ & $"$ & media & muy alta & 0.5 & $"$ & $\begin{array}{l}\mathrm{c}, \mathrm{t}, \mathrm{m} \\
\mathrm{fl}\end{array}$ \\
\hline & & 3 & $\begin{array}{l}\text { cantos } \\
\text { rodados }\end{array}$ & $"$ & 22 . & $"$ & muy alta & lenta & $"$ & $"$ & $\mathrm{ta}, \mathrm{fl}$ \\
\hline & & 4 & $\begin{array}{l}\text { islote con } \\
\text { troncos }\end{array}$ & $"$ & $"$ & $"$ & alta & $"$ & 0.2 & troncos & $\begin{array}{l}\mathrm{c}, \mathrm{ta}, \\
\mathrm{fl}\end{array}$ \\
\hline & & 5 & $\begin{array}{l}\text { márgen } \\
\text { del río }\end{array}$ & $"$ & $"$ & $"$ & media & $"$ & 0.5 & ígneo & $\mathrm{ta}, \mathrm{fl}$ \\
\hline & & 6 & plataforma & $"$ & $"$ & $"$ & alta & $"$ & $"$ & $"$ & $\mathrm{fl}$ \\
\hline
\end{tabular}


CUADRO 1. Continuación.

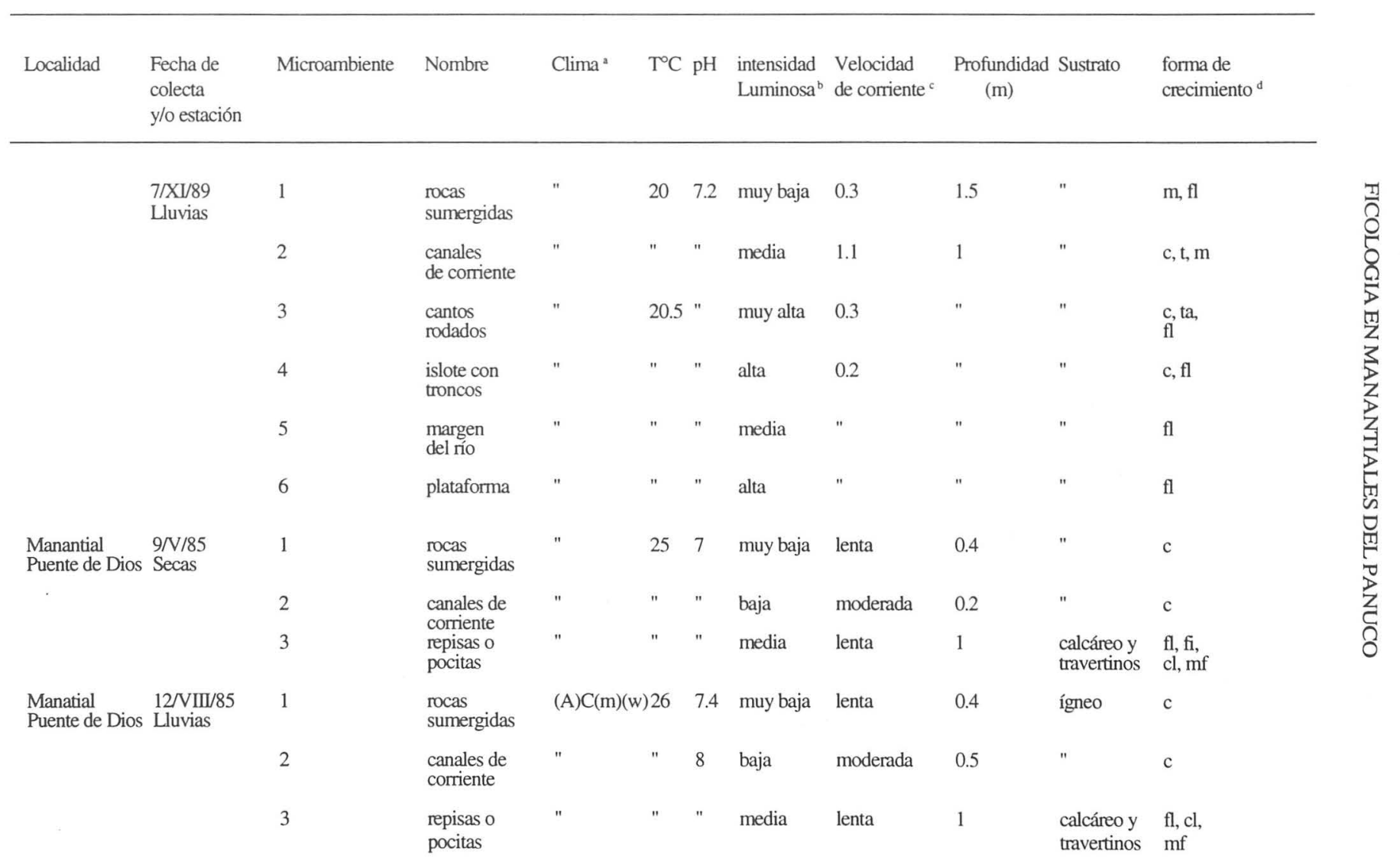


CUADRO 1. Continuación.

\begin{tabular}{|c|c|c|c|c|c|c|c|c|c|c|c|}
\hline Localidad & $\begin{array}{l}\text { Fecha de } \\
\text { colecta } \\
\text { y/o estación }\end{array}$ & Microambiente & Nombre & Clima & $\mathrm{T}^{\circ} \mathrm{C}$ & $\mathrm{pH}$ & $\begin{array}{l}\text { intensidad } \\
\text { Luminosa }^{b}\end{array}$ & $\begin{array}{l}\text { Velocidad } \\
\text { de corriente }{ }^{c}\end{array}$ & $\begin{array}{l}\text { Profundidad } \\
\text { (m) }\end{array}$ & Sustrato & $\begin{array}{l}\text { forma de } \\
\text { crecimiento }\end{array}$ \\
\hline & $\begin{array}{l}14 / V / 89 \\
\text { Secas }\end{array}$ & 1 & $\begin{array}{l}\text { rocas } \\
\text { sumergidas }\end{array}$ & $"$ & $"$ & 7.8 & muy baja & 0.15 & 0.4 & ígneo & c \\
\hline & & 2 & $\begin{array}{l}\text { canales de } \\
\text { corriente }\end{array}$ & $"$ & $"$ & $"$ & baja & 0.2 & 0.3 & $"$ & c \\
\hline & & 3 & $\begin{array}{l}\text { repisas o } \\
\text { pocitas }\end{array}$ & $"$ & $"$ & $"$ & media & 0.13 & 1 & $\begin{array}{l}\text { calcáreo y } \\
\text { travertinos }\end{array}$ & $\begin{array}{l}\mathrm{c}, \mathrm{cl} . \\
\mathrm{mf}\end{array}$ \\
\hline
\end{tabular}

a Según Instituto Nacional de Estadística Geografia e Informática, 1985.

A w1.- Tipo cálido subhúmedo con lluvias en verano, \% de lluvia invernal entre 5 - 10.2 (intermedio en cuanto a humedad).

(A) C (m) (w).- Tipo semicálido húmedo con abundantes lluvias en verano. \% de lluvia invernal menor de 5.

b Según Johansson (1982): insolación muy alta $=>80 \%$ del horizonte visible; alta $=60-80 \%$; media $=40-60 \%$; baja $=20-40 \%$ y muy baja $=<20 \%$.

c Según Johansson (1982): Para las colectas anteriores a 1989, $<0.1 \mathrm{~m} / \mathrm{seg}=$ muy baja (casi estancada); 0.1-0.35 m/seg $=$ lenta; $0.35-0.6 \mathrm{~m} / \mathrm{seg}=\mathrm{moderada} ; 0.6-0.85 \mathrm{~m} /$ seg=alta $y>0.85 \mathrm{~m} / \mathrm{seg}=$ muy alta.

dFormas de crecimiento, Holmes y Whitton (1981) y Tavera y González-González $(1990): \mathrm{c}=\cos t r a, t=t u f o s, \mathrm{fl}=$ flóculo filamentoso, ta $=$ tapete, $\mathrm{mf}=$ masa de filamentos flotantes, $\mathrm{m}=$ crecimientos sobre musgo, $\mathrm{cl}=$ colchón y fi = filamentos filiformes. 
CUADRO 2. Total de especies y su porcentaje por clase de los manantiales de estudio.

$\begin{array}{r}\text { Clase } \quad \begin{array}{r}\text { Número y porcentaje } \\ \text { de especies }\end{array} \\ \hline\end{array}$

$\begin{array}{llr}\text { Manantial } & \text { Cyanophyceae } & 7(29.16 \%) \\ \text { Choy } & \text { Chlorophyceae } & 4(16.66 \%) \\ & \text { Rhodophyceae } & 2(8.33 \%) \\ & \text { Bacillariophyceae } & 11(45.83 \%) \\ \text { Manantial } & \text { Cyanophyceae } & \\ \text { Huichihuayan } & \text { Chlorophyceae } & 13(29.54 \%) \\ & \text { Rhodophyceae } & 8(18.18 \%) \\ & \text { Xanthophyceae } & 2(4.54 \%) \\ \text { Bacillariophyceae } & 1(2.27 \%) \\ \text { Manantial } & \text { Cyanophyceae } & 20(45.45 \%) \\ \text { Puente de Dios } & \text { Chlorophyceae } & 10(27.02 \%) \\ & \text { Rhodophyceae } & 4(10.81 \%) \\ & \text { Xanthophyceae } & 3(8.10 \%) \\ & \text { Bacillariophyceae } & 1(2.70 \%) \\ & & 19(51.35 \%)\end{array}$

y de Blenothrix ganeshii, Xenoccocus sp., Cladophora glomerata, Oedogonium sp. 3 en el microambiente 3 del manantial Puente de Dios.

\section{DISCUSIÓN}

Al analizar la composición de especies de la zonas delimitadas en cada localidad se encontró que las zonas 1 y 2 de Puente de Dios presentaron básicamente las mismas especies, por lo que se consideró como un mismo microambiente. El resto de las zonas de cada una de las localidades correspondió con diferentes conjuntos de especies por lo que las consideramos como microambientes distintos, lo que valida la utilidad de emplear la forma de crecimiento en relación con parámetros ambientales, para reconocer microambientes. El escaso porcentaje de especies comunes a las tres localidades en todas las colectas, pareciera indicar una escasa similitud en relación a la ficoflora de los tres manantiales. Sin embargo, la mayor diversidad de especies, que se presentó en la localidad Huichihuayan, estuvo directamente relacionada con un mayor número de microambientes (cuadro 1), es decir, con condiciones ambientales no presen- 
CUADRO 3. Lista de especies por colecta en los microambientes del manantial Choy.

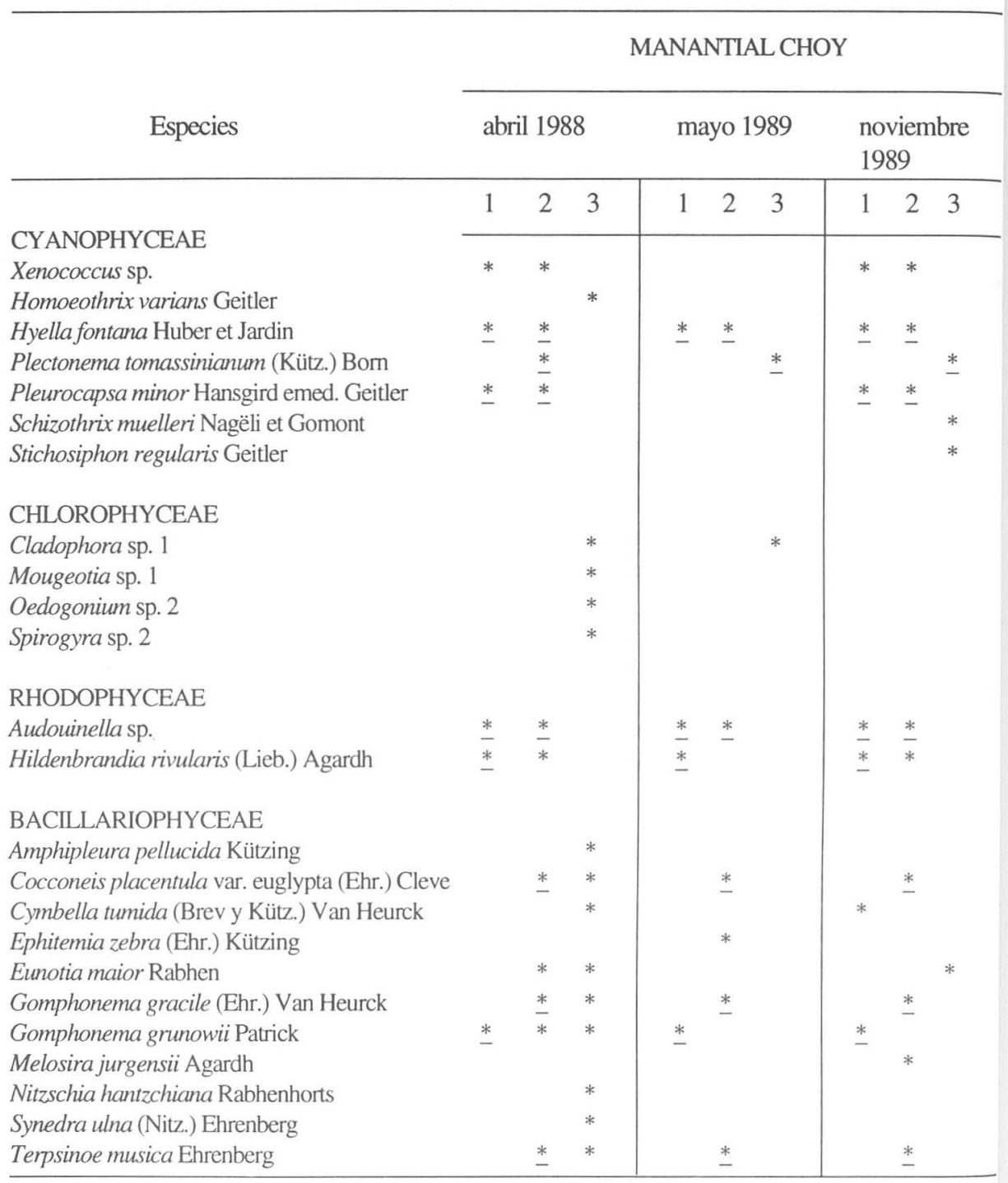

Choy.- Microambientes: 1 canales de corriente dentro de la caverna; 2, cantros rodados dentro de la caverna; 3 , rocas emergentes fuera de la caverna.

*.- especie que se presentó de forma recurrente espacial y temporalmente en el manatial. 


\section{CUADRO 4. Lista de especies por colecta en los microambientes del manantial Huichihuayan.}

\section{MANANTIAL HUICHIHUAYAN}

\section{ESPECIES}

marzo 1984

mayo 1985

noviembre 1989

CYANOPHYCEAE

Anabaena sp.

Chamaesiphon confervicola Brown in Rabhen

Chrooccocus turgidus Nägeli

Chamaecalix sp.

Xenococcus sp.

Homoeothrix juliana (Menegh.) Krichn

Homoeothrix varians Geitler

Merismopedia glauca (Ehr.) Nägeli

Merismopedia sp.

Myxosarcina choococcoides Geitler

Phormidium autumnale (Ag.)J. Schmidt

Pleurocapsa minor Hansgird emed. Geitler

Schizothrix muelleri Nagëli et Gomont

CHLOROPHYCEAE

Cladophora sp. 2

Closterium moniliferum (Bory) Ehrenberg

Mougeotia sp. 2

Oedogonium sp. 1

Scenedesmus acutus var. alternans Meyen

Scenedesmus ecomis (Ehr.) Chod

Spirogyra sp. 1

Stigeoclonium tenue Kützing

RHODOPHYCEAE

Audouinella sp.

Hildenbrandia rivularis (Lieb.) Agardh

XANTHOPHYCEAE

Vaucheria sp.

BACILLARIOPHYCEAE

Achnanthes inflata (Kütz.) Grun

Amphipleura pellucida Kützing

Amphora ovalis Kützing

Campylodiscus noricus var. hebernica Ehrenberg

Cocconeis placentula var. euglypta (Ehr.) Cleve

Cymbella tumida (Breb y Kütz.) Van Heurck

Denticula tenuis Kützing

Gomphonema acuminatum Ehrenberg

Gomphonema gracile (Ehr.) Van Heurck

Gyrosima acuminatum (Kutz.) Rabh

Gyrosima scalproides (Rabh.) Cleve

Melosira jurgensii Agardh

Melosira undulata (Ehr.) Kützing

Navicula mutica var. undulata (Hilse) Grun

Navicula viridula var. rostellata (Kütz.) Cleve

Nitzschia hantzschiana Rabhenhorts

Nitszchia sinuata W. Smith

Surirella linearis var. constrictta W.Smith

Synedra ulna (Nitz.) Ehrenberg

Terpsinoe musica Ehrenberg
$123456 \quad 123456123456$

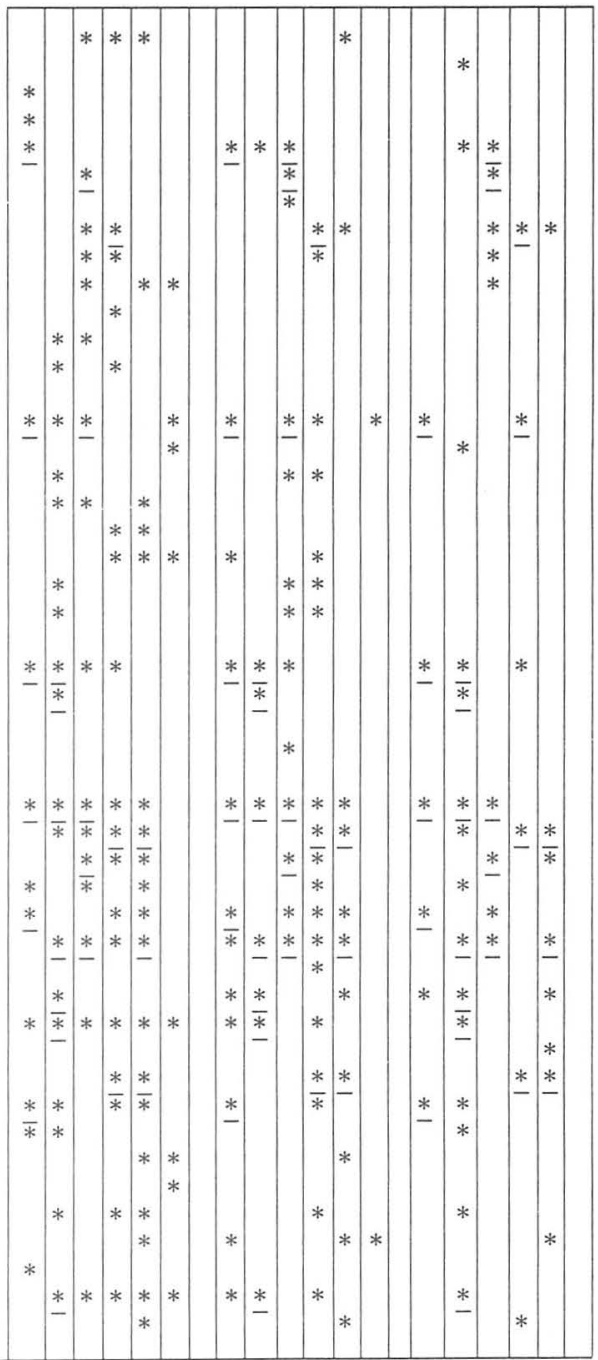

Huichihuayan.- Microambientes: I, rocas emergentes en la caverna; 2 , canal de corriente fuera de la caverna; 3 , cantos rodados fuera de la caverna en el centro del cauce; 4 , Islote con troncos fuera de la caverna; 5 , márgen del río fuera de la caverna; 6 , plataforma de rocas sumergidas al márgen del cauce fuera de la caverna.

* especie que se presentó de forma recurrente espacial y temporalmente en el manantial. 
CUADRO 5. Lista de especies por colecta en los microambientes del manantial Puente de Dios.

MANANTIAL PUENTE DE DIOS

ESPECIES

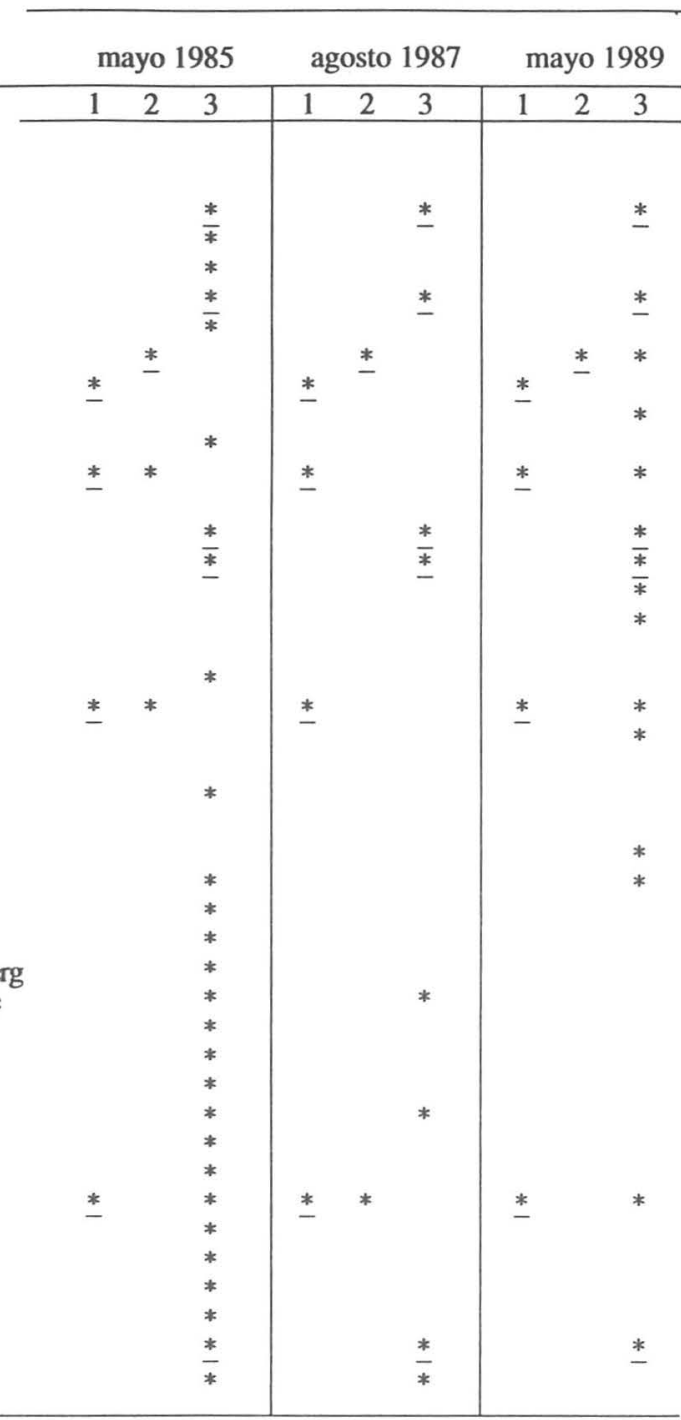

\section{CYANOPHYCEAE}

Blenothrix ganeshii Watanabe et Komarek

Chrooccocus turgidus Nägeli

Chamaecaliyx sp.

Xenococcus sp.

Homoeothrix juliana (Menegh.) Krichn

Homoeothrix varians Geitler

Hyella fontana Huber et Jardin

Merismopedia glauca (Ehr.) Nägeli

Plectonema tomassinianum (Kütz) Bom

Pleurocapsa minor Hansgird emed. Geitler

CHLOROPHYCEAE

Cladophora glomerata (Linn.) Kützing

Oedogonium sp.3

Scenedesmus acutus var. alternans Meyen

Scenedesmus ecomis (Ehr.) Chod

RHODOPHYCEAE

Audouinella sp.

Hildenbrandia rivularis (Lieb) Agardh

Thorea riekei $\mathrm{Bishoff}$

XANTHOPHYCEAE

Vaucheria sessilis De Candolle

BACILLARIOPHYCEAE

Achnanthes inflata (Kütz.) Grun

Amphipleura pellucida Kützing

Amphora ovalis Kützing

Biddulphia laevis Ehrenberg

Campylodiscus noricus var. hebernica Ehrenberg

Cocconeis placentula var. euglypta (Ehr.) Cleve

Cymbella mexicana (Ehr.) Cleve

Cymbella minuta var. silesiaca Bleisch y Rabh

Gomphonema acuminatum Ehrenberg

Gomphonema gracile (Ehr.) Van Heurck

Gyrosigma scalproides (Rabh.) Cleve

Navicula radiosa Kützing

Nitzschia hantzschiana Rabhenhorts

Pinnularia maior (Kütz.) Rabhenhorts

Rhopalodia gibba (Ehr.) Müller

Surirella robusta Ehrenberg

Synedra rumpens var. scotica Grun

Synedra ulna (Nitz) Ehrenberg

Terpsinoe musica Ehrenberg

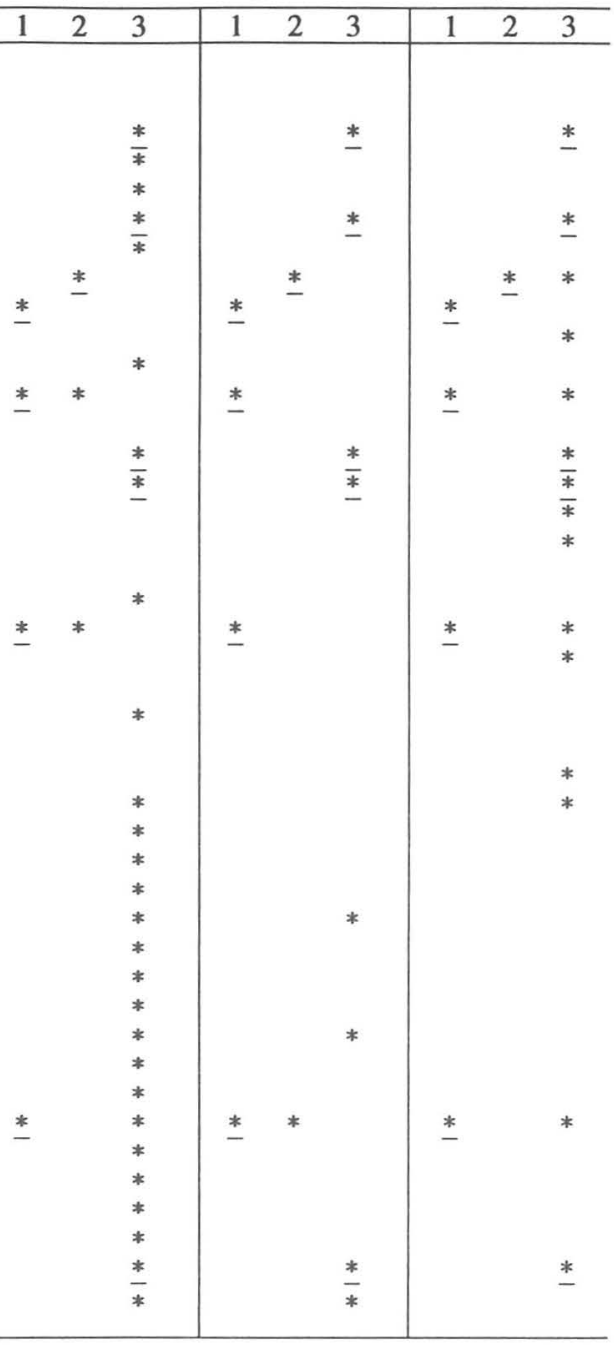

Puente de Dios.- Microambientes: 1, pequeñas rocas sumergidas en la caverna; 2, canales de corriente fuera de la caverna; 3 , repizas o pocitas fuera de la caverna.

* -Especie que se presentó de forma recurrente espacial y temporalmente en el manantial. 
tes en las otras localidades. Tal es el caso de los microambientes 3 y 4 , los más diversos de todos los microambientes revisados, con una rica comunidad metafítica (Round, 1984), relacionada con condiciones ambientales de alta intensidad luminosa, sustrato de cantos rodados y poco movimiento de agua, lo que posibilita la deposición de detritos, por lo que podríamos considerar que estas comunidades están más relacionadas con las comunidades del inicio del río, más que con las de los manantiales. De la misma manera, en los manantiales Choy y Puente de Dios encontramos que la mayor diversidad de especies observada se refiere al microambiente 3 , los cuales presentan condiciones ambientales más relacionados con el inicio de los ríos que con la de los manantiales.

En cuanto a las asociaciones comunes a los tres manantiales cabe destacar a Hildenbrandia rivularis y Audouinella sp. (fig.3). H. rivularis, a pesar de ser cosmopolita, es una especie con plasticidad adaptativa reducida, que creció solo en condiciones muy particulares, que incluyen agua corriente rica en nutrientes e intensidades luminosas muy bajas (Starmach, 1969a y 1969b). Audouinella sp. es una especie que formó tufos sobre rocas y que al igual que $H$. rivularis se desarrolló dentro de cavernas o zonas muy sombreadas, siempre en relación a velocidades de corriente alta o muy alta de más de $1.3 \mathrm{~m} / \mathrm{seg}$, como es el caso del microambiente 1 de Choy o el microambiente 2 de Huichihuayan. Además, se ha observado que $H$. rivularis tiene una taza de crecimiento muy lento, por lo que generalmente se encuentran costras de pequeño tamaño de unos cuantos centímetros de diámetro. Dicha especie la encontramos formando costras pequeñas a lo largo de ríos en la Huasteca Potosina, en lugares con baja iluminación, en la porción inferior de rocas o creciendo por debajo de musgos acuáticos. Sin embargo, en el caso de los tres manantiales estudiados $H$. rivularis formó crecimientos que alcanzar hasta $2 \mathrm{~m}$, en particular el microambiente 1 del Choy y microambientes 1 y 2 de Puente de Dios. Estos crecimientos profusos parecen estar relacionados con las características de estabilidad de los manantiales. En el caso de Audouinella sp. cabe resaltar que Sheath y Hambrook (1990) reportan la presencia de esta especies en velocidades de corriente que van de 0.4 a $0.88 \mathrm{~m} / \mathrm{seg}$, intervalo muy por debajo de los registrados en el presente trabajo.

Otra asociación común entre los manantiales fué Hildenbrandia rivularis, Hyella fontana (fig.3) y Pleurocapsa minor, formas costrosas que se desarrollan epilíticamente dentro de cavernas en relación a baja intensidad luminosa y velocidad de corriente muy baja o lenta de menos de $0.3 \mathrm{~m} / \mathrm{seg}$, condiciones encontradas en el microambiente 2 del Choy y microambientes 1 y 2 de Puente de Dios, aunque en la primera se registró una velocidad de corriente moderada.

Aunque las especies que dan la estructura a las formas de crecimiento algales en los manantiales reportados para este trabajo, no se encuentran exclusivamente en el ambiente manantial, en conjunto podemos afirmar que forman asociaciones que se desarrollan bajo condiciones ambientales muy precisas, las cuales estan presentes en los manantiales estudiados. 

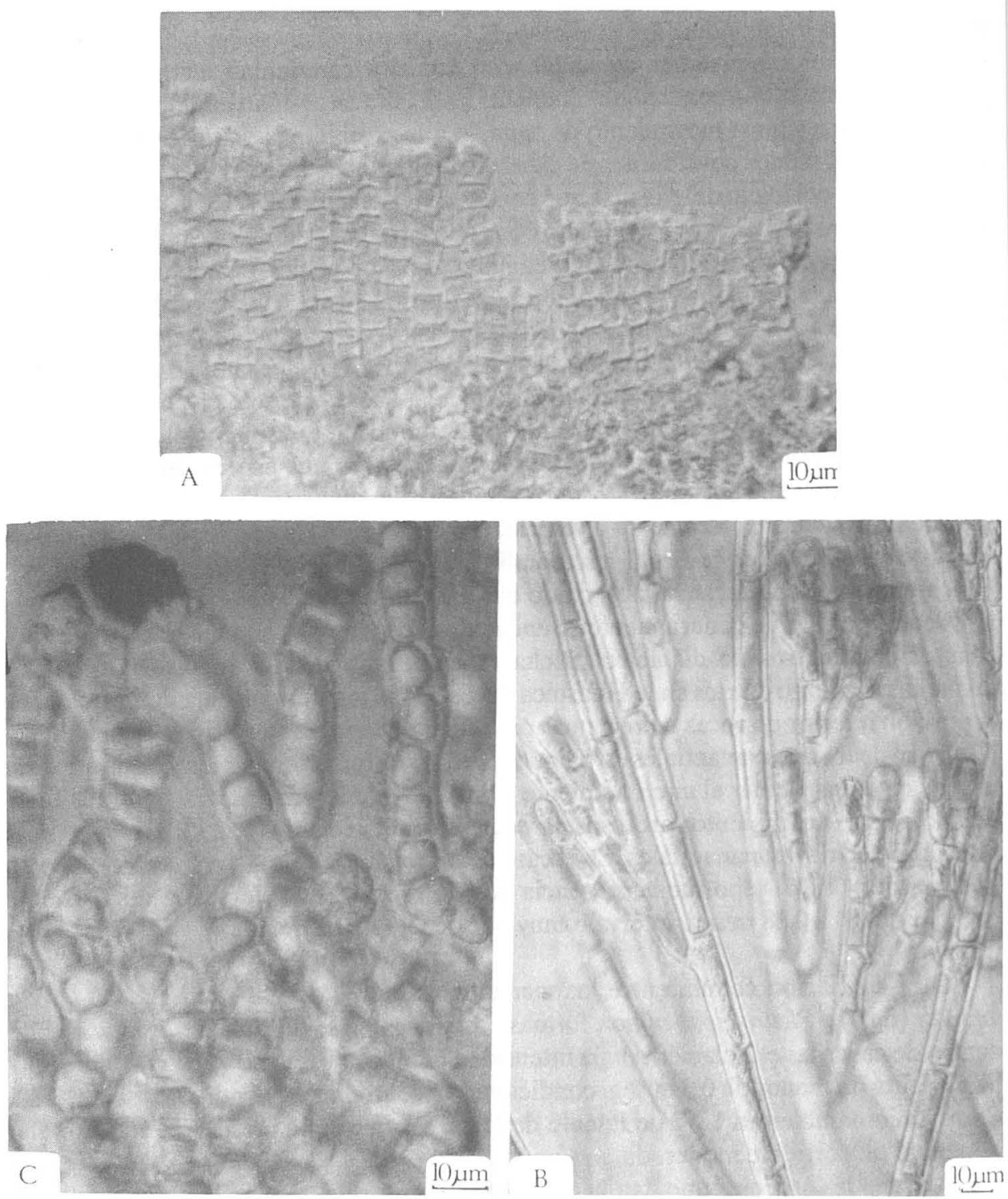

Fig.3 Especies recurrentes en los tres manantiales de estudio: A. Sección transversal del talo costroso de Hildenbrandia rivularis. B. Talo filamentoso de Audouinella sp. C. Sección transversal del talo costroso de Hyella fontana. 
Resulta importante ampliar el estudio de las algas de manantiales a otras regiones con diferente fisiografía y origen geológico para demostrar que la estabilidad de los factores: iluminación, velocidad de corriente y sustrato, son elementos determinantes en la presencia de las asociaciones características de este ambiente.

AgradECIMIENTOS. A la M. en C. Elisa Serviere Zaragoza del Laboratorio de Ficología, Facultad de Ciencias, UNAM, por su orientación y sugerencias para la realización de este trabajo. El trabajo se desarolló como parte del proyecto IN203089 financiado por la Dirección General de Asuntos del Personal Académico (DGAPA), UNAM.

\section{LITERATURA CITADA}

ANAGNOSTIDIS, K. y J. KOMAREK. 1985. Modern approach to the classification system of Cyanophytes. 1. Introduction. Arch. Hydrobiol. Suppl. 71,1-2, Algological Studies 38-39:291-292.

ANAGNOSTIDIS, K. y J.KOMAREK. 1988. Modern approach to the classification system of Cyanophytes.

3. Oscillatoriales. Arch. Hydrobiol. Suppl. 80,1-4, Algological Studies 50-53:327-472.

BISHOFF, H.W. 1965. Thorea riekei sp. nov. and related species. J. Phycol. 1:111-117.

BOURRELLY, P. 1968. Les algues d'eau douce. Initiation à la Systématique. Les algues jaunes et breunes. Ed. N. Boubée et Cie, París, T.II. 517p.

BourRelLy, P. 1970. Les algues d'eau douce. Initiation à la Systématique. Les algues bleues et rouges. Ed. N. Boubée et Cie, París, T.III. 512p.

Bourrelly, P. 1972. Les algues d'eau douce. Initiation àla Systématique. Les algues vertes. Ed. N. Boubée et Cie, París, T.I. 572p.

DARY, L.J.y H.N. WAYNE. 1968. Chlorophycean Algae from limestone Spring. Amer. J. Bot. 55(2):205213.

DESIKACHARY. T.V. 1959. Cyanophyta. Indian Council Agricultural Research, New Delhi, India, 686p.

GEITLER, L. 1932. Cyanophyceae. En: L. Rabenhort's. KryptogamenFlora von Deutschland Osterreich und der Scheiz. Akadesmische Verlagsgasellchaft, Leipzig, 1196p.

GERMAin, H. 1981. Flore des Diatomées. Diatomophycées. Societé Nouvelle des Editions Boubée, París, $444 \mathrm{p}$.

GOLUBIC, S. 1967. Algenvegetation der Falsen die Binnengewasser E. Scheweizerbart'sche Verlagsbuchhandlung (Nägele u. Obermiller). Stuttgard, Germany. 183p.

Holmes, N.T.H. y B.A. WhITTON. 1981. Phytobentos of the river tees and its tributaries. Freshwater Biology 11:139-163.

HutCHINSON, G.E. 1967. A treatise on limnology. Introduction to lake biology and the limnoplankton. J.Wiley \& Sons, Inc. New York, London. v. 2. 1115p.

HYNES, H.B. 1970. Ecology of running waters. 4a. ed. University of Toronto, Press. G.B. The Chaucer Press Ltd Bungag, Suffolk. Great Britain. 518p.

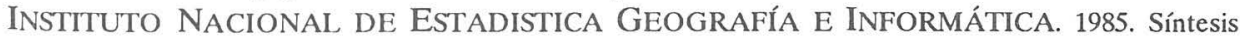
Geográfica del estado de San Luis Potosí. Secretaría de Programación y Presupuesto, México. 186p.

ISLAM, A.K. 1963. A revision of the genus Stigeoclonium. Verlag von J. Cramer, Weinheim, Germany. 164p. 
ISRAELSON, G. 1942. The Freshwater florideae of Sweden. Symbolae Botanicae Upsalienses VI. 130p.

JOHANSSON, C. 1982. Attached algal vegetation in running waters of Jämtland, Sweden. Acta Phytogeogr. Suec. 71:1-80.

KANN, E. y J. KOMAREK. 1970. Systematishokologische Bemerkungen zuden arten des Formenkreises Phormidium autumnale. Hydrologie 32(2):495-519.

KYLIN, H. 1956. Die gattungen der Rhodophyceen. CWK Gleerups Forlag Lund. Schweden. 673p.

KOMAREK, J. y E.KANN. 1973. Zur taxonomie und okologie der gattung Homoeothrix. Arch. Protistenk 115:173-233.

KOMAREK, J.y K. ANAGNOSTIDIS. 1986. Modern approach to the classification system of Cyanophytes. 2-Chroococcales. Arch. Hydrobiol. Suppl. 73,2, Algological Studies 43:157-226.

KOMAREK, J. y L. KOVACIK. 1987. Revision of several species of the genus Homoeothrix (Cyanophyta). Preslia, Praha 59:229-292.

KOMAREK, J.y K. ANAGNOSTIDIS. 1989. Modern approach to the classification system of Cyanophytes. 4-Nostocales. Arch. Hydrobiol. Suppl. 82,3, Algological Studies 56: 247-345.

MARGALEFF, R. 1977. Ecología. Ed.Omega. Barcelona, España. 951p.

ORTEGA, M.M. 1984. Catálogo de algas continentales recientes de México. Coordinación de la Investigación Científica. Universidad Nacional Autónoma de México, México. 566p.

PATRICK, R. y CH.W. REIMER. 1966. The diatoms of the United States. Monographs of the Academy of Natural Sciences of Philadelphia, Pennsylvania. v. 1. No. 13. 688p.

PATRICK, R. y CH.W. REIMER. 1975. The diatoms of the United States. Monographs of the Academy of Natural Sciences of Philadelphia, Pennsylvania. v. 2. No. 13. 213p.

ROUND, F.E. 1973. The biology of the Algae. 2a. ed. St. Martin's Press. New York, 278p.

ROUND, F.E. 1984. The ecology of the Algae. 2a. ed. Cambridge University Press, Great Britain. 653p.

SAMANO, A.B. 1932. Contribución al conocimiento de las algas de las fuentes termales de Ixtapan de la Sal. Anales. Inst. Biol. Univ. Nac. México 3:49-50.

SARODE, P.T.y N.D. KAMAT. 1984. Freshwater Diatoms of Maharashtra. Saikripa Prakashan, Aurangabad (Maharashtra). 338p.

SECRETARÍA DE RECURSOS HIDRÁUliCOS. 1971. Jefatura de Irrigación y Control de Ríos. Dirección de Hidrología. México. Bol. Hidrol. 44:(1-03.11).

SheATH, R.G. y J.A. HAMBROOK. 1990. Freshwater ecology. En: Cole, K.M. y Sheath, R.G.(Edrs.). Biology of the red algae. Cambridge University Press. New York. 517p.

SOKOLOFF, D. 1936. Análisis hidrobiológico del manantial de la "Mora" de Actopan, Hgo. Anales. Inst. Biol. Univ. Nac. México 7:287-303.

STARMACH, K. 1966. Flora Slodkowodna Polski. Tom. 2: CyanophytaGlaucophyta. Polska Akademia Nauk. WarszawaKraków. Poland. 807p.

STARMACH, K. 1969. Flora Slodkowodna Polski. Tom. 2: ChlorophytaOedogoniales. Polska Akademia Nauk. WarszawaKraków. Poland. 659p.

STARMACH, K. 1969a. Hildenbrandia rivularis and asociating it Algae in the Stream Cedronka near Wejherowa (Gdanskvoivodo). Fragmenta Flor. et Geobotanica (15)3:387-398.

STARMACH, K. 1969b. Growth of talli and reproduction of the red alga Hildenbrandia rivularis (Liebm.) J. Ag. Acta Soc. Bot. Polon. 38(3):523-533.

StarmaCH, K. 1977. Flora Slodkowodna Polski. Paeophyceae Rhodophyceae. T.4. Polska Akademia Nauk. WarszawaKrakow. Poland. 445p. 
TAVERA S.,R.L. y J. GONZÁLEZ-GONZÁLEZ. 1990. Caracterización ficoflorística de los paredones de la Sierra de Juárez, Oaxaca. Importancia de las formas de crecimiento algales en la tipificación de un ambiente. Bol. Soc. Bot. México 50:121-133.

TranSEAU, N.E. 1951. The Zygnemataceae. Graduate School Monographs. University of Ohio State, Columbus. 328p.

VAN DEN HOEK. 1963. Revision of the European species of Cladophora. E.J.Brill. Leiden, Netherlands. 247 p.

VASILIEV, Y.M. y M.S. ARABADZHI. 1981. Geología general e histórica. Ed. Mir, Moscú. 404p.

VENTAKATAMARAN. 1961. Vaucheriaceae. Indian Agricultural Research Institute, New Delhi. 111p.

WATANABE,M. y J. KOMAREK. 1989. New Blenothrix-species (Cyanophyceae/Cyanobacteria) from Nepal. Bull. Nat. Sci. Mus. Tokyo 15(3):67-79.

WEST, W. y G.S. West. 1904. A monograph of the British Desmidiaceae. Printed for the Ray Society. London. v. 1.300p.

WHITFORD, L.A. 1956. The communities of Algae in the springs and spring stream of Florida. Ecology 37(3):433-442. 